\title{
Use of Additive Manufacturing (AM) to Fabricate Injection Molding Inserts
}

\author{
Jorge Rodriguez, $\mathrm{PhD}$ \\ Western Michigan University, Department of Engineering Design, Manufacturing, and Management Systems \\ Kalamazoo, MI, USA, jorge.rodriguez@wmich.edu
}

\begin{abstract}
Using Additive Manufacturing (AM) to produce inserts for injection molding of parts used for rapid quote can drastically reduce costs and time frame. Prototype injection molding currently can take weeks and thousands of dollars to develop and manufacture, making $A M$ as a very viable option. Functional prototypes of mold inserts were developed and analyzed with $C A D$ software, then printed on a $3 D$ printer, and tested using industrial plastic injection molding equipment. Calculations for cooling and mold compression were used to develop guidelines for future parts. Measurements were taken on the molded parts in order to validate calculations and dimensions. This methodology is beneficial to industry because a company can rapidly and efficiently produce functional parts for quoting for prospective clients.
\end{abstract}

Keywords: Additive Manufacturing, 3DP, Injection Molding.

Digital Object Identifier (DOI):

http://dx.doi.org/10.18687/LACCEI2016.1.1.345

ISBN: 978-0-9822896-9-3

ISSN: 2414-6390

$14^{\text {th }}$ LACCEI International Multi-Conference for Engineering, Education, and Technology: "Engineering Innovations for Global Sustainability", 20-22 July 2016, San José, Costa Rica. 


\title{
Use of Additive Manufacturing (AM) to Fabricate Injection Molding Inserts
}

\author{
Jorge Rodriguez, $\mathrm{PhD}$ \\ Western Michigan University, Department of Engineering Design, Manufacturing, and Management Systems \\ Kalamazoo, MI, USA, jorge.rodriguez@wmich.edu
}

\begin{abstract}
Using Additive Manufacturing (AM) to produce inserts for injection molding of parts used for rapid quote can drastically reduce costs and time frame. Prototype injection molding currently can take weeks and thousands of dollars to develop and manufacture, making $A M$ as a very viable option. Functional prototypes of mold inserts were developed and analyzed with CAD software, then printed on a $3 D$ printer, and tested using industrial plastic injection molding equipment. Calculations for cooling and mold compression were used to develop guidelines for future parts. Measurements were taken on the molded parts in order to validate calculations and dimensions. This methodology is beneficial to industry because a company can rapidly and efficiently produce functional parts for quoting for prospective clients.
\end{abstract}

Keywords: Additive Manufacturing, 3DP, Injection Molding.

\section{INTRODUCTION}

Plastic injection molding is a technology that is widely used in industry nowadays mainly because of its wide range of applicability. Unfortunately, even for prototypes or short-runs, it can take several weeks and thousands of dollars to develop and have a product manufactured. Prototype injection molds are used to produce short runs (i.e., less than a hundred) of functional parts, or to have an initial run that will help in quoting customers' requirements. Injection molds are primarily manufactured using tool steels which can withstand the high temperature environment as well as well as mechanical and thermal fatigue. Using Additive Manufacturing (i.e., 3-D Printing) to produce made-to-size inserts for injection molds has the potential to drastically reduce those costs and time of production.

It is important to have a very good understanding of the concepts and methods in injection molding technology when developing additively manufactured plastic mold insert. Several variables need to be taken into consideration during injection molding process, e.g., compression pressures, hold time, and cooling. Compression directly affects injection molded parts in terms of quality, due to the part deformation caused by volumetric shrinkage. Volumetric shrinkage is the reduction of molecule size in a particular plastic when changing between molten and solid states. Compression also affects the proper sealing of the mold halves, flashing is likely to occur without a proper seal. These are some of the aspects that were

Digital Object Identifier (DOI): http://dx.doi.org/10.18687/LACCEI2016.1.1.345 ISBN: 978-0-9822896-9-3

ISSN: $2414-6390$ considered in this study when specifying a mold insert that is placed into a steel mold.

Functional insert molds were developed and analyzed with CAE software packages for modeling, stress analysis, and injection molding simulation. The developed inserts were fabricated on a 3D printer, and tested using an industrial plastic injection molding machine. Calculations for cooling and mold compression were used to develop guidelines for future parts, and specific dimensions were taken to validate the molded parts. Several sets of the 2-part inserts were fabricated and tested, and results show the potential of this type of process for specific applications. Therefore, it is possible to have a benefit with the use of additive manufacturing for short runs and rapid quoting of plastic injection molded parts.

\section{BACKGROUND}

Additive Manufacturing (AM) is the process of additively producing a 3D part through a layered process. AM is a field that has exploded with technologies and applications. Among those technologies is the concept of 3DP (3D Printing), which is a technology applied by several of the AM machines in use today [1]. With a specific field of applications in mind, AM can be considered for rapid prototyping, rapid tooling, or rapid quoting. Recent developments have improved tolerances, capacity for thinner layers, and wider material. Although the 3D printing technology, particularly when referring to FDM (Fused Deposition Modeling) is well understood, there is always room for improvement [2]. But numerous technological developments nowadays are in the area of materials used in 3D printing. The selected material will depend on the available 3DP machine and the particular application [3]. The overall goal in these developments is to move from the rapid prototype to a functional part.

A company that has been a pioneer in developing 3D printing technology and materials is Objet (now part of Stratasys Corp) [4]. This company applies polyjet technology that is similar to an inkjet printer using a print head that instead of printing water-based ink, lays down thin layers of photopolymer material. These machines have the capability to print in layers as thin as 15 microns (0.0063 inches); this is thinner than a human hair. By being able to apply material at such thin layers, higher resolution parts (i.e., mold inserts in our

$14^{\text {th }}$ LACCEI International Multi-Conference for Engineering, Education, and Technology: "Engineering Innovations for Global Sustainability”, 20-22 July 2016, San José, Costa Rica. 
case, with small tolerances) are a real possibility. Access to a Stratasys (Object) machine was available for this project [5].

Injection molding is the process of injecting molten plastic material into a mold cavity with the proper injection parameters to produce a functional part [6]. Plastic injection molds are primarily manufactured using tool steels which can withstand the high temperature environment. It is important to consider the parameters than are usually specified in the regular injection molding process, so that appropriate models and materials are selected in the 3DP process. Several variables need to be taken into consideration during injection molding, e.g., compression pressure, hold time, and cooling. Compression affects injection molded parts in terms of quality, due to part deformation caused by volumetric shrinkage. Volumetric shrinkage is the reduction of molecule size in a particular plastic when changing between molten and solid states. Compression also affects the proper sealing of the mold halves, without a proper seal flashing is likely to occur. This study considers the fabrication of mold inserts to be placed into a steel mold for the fabrication of plastic parts. Thus, the compression ratios then needed to be studied and adjusted in the design of the mold insertsfor this study. These mold inserts will produce the benefit of reduced lead times and overall cost of functional parts for short runs and rapid quoting $[7,8,9,10]$.

The goal of the project is to determine the feasibility of utilizing additive manufacturing for short runs, as well as quoting of functional injection molded parts. There are reports that have dealt with this issue of producing injection molded parts with 3DP inserts [11,12], with many of the projects being a trial-and-error approach producing couple of parts. The potential for larger benefit is there but there needs to be dedicated efforts in this application. The objective of this project is to perform design and testing for additive manufactured mold insert design. The design of the insert is determined based on desired molded part, mold material, cooling channels, injection material, and mold life. Initial design guidelines for some geometry features are studied.

\section{METHODOLOGY}

The steps taken to complete this feasibility study were:

- Research process and material properties

- Create initial/modified designs

- Determine injection parameters

- Perform Moldflow and Finite Element Analysis

- Define cooling channel

- Do testing of printed inserts.

Some of the specific developments carried out are:

Material Properties. Different material properties were obtained for the temperature-resistant materials available for the manufacturer. This information helped understand how the materials were going to behave in the injection molding process. Some properties were provided by the supplier, but for

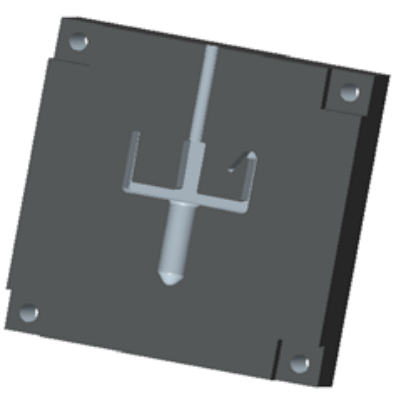

(a) Initial Design

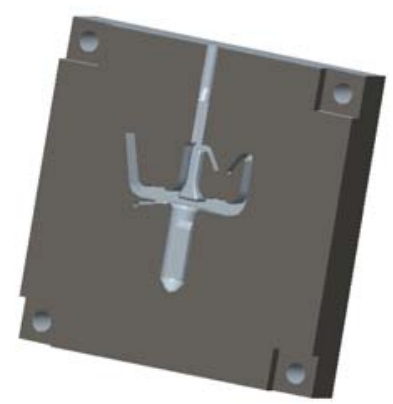

(b)Modified Design

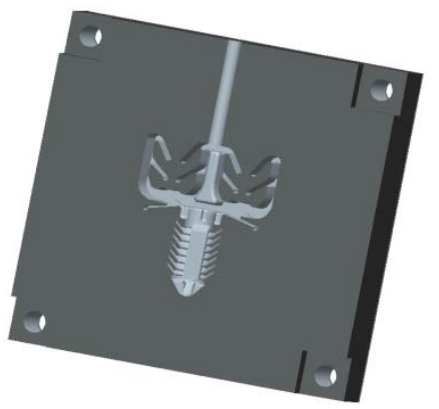

(c)Final Design

Figure 1. Progression in Mold Insert Design

some other properties the information was not available, and further research and testing was required to have approximated values. The material being used is VeroGray, with a Modulus of Elasticity of 3,000 $\mathrm{MPa}$, compared to aluminum which has a modulus of 70,000 $\mathrm{MPa}$. The heat deflection temperature of VeroGray is at $250^{\circ} \mathrm{C}\left(120^{\circ} \mathrm{F}\right)$, compared to aluminum which is $2,192^{\circ} \mathrm{C}\left(1,200^{\circ} \mathrm{F}\right)$. Poisson's Ratio was determined by looking at similar materials (i.e., Fullcure 720), and by performing compression testing.

Mold Design Progression. In designing the mold insert it was decided to do a progression of designs. With this approach, the initial design includes basic features and progressively includes more features and higher degree of complexity. The reason this approach was followed is to help in the understanding of the effects of simple geometries and build upon it. This approach helped as well in getting a better understanding of the molding process and deciding injection parameters. Three primary designs were developed for experimentation in this project; the designs went through a progression from basic to more complex (Fig. 1).

The first design was made up of simplified features from the final part. The first design (Fig. 1a) has two fingers that protrude upwards with a curved section to represent one of the eight curved fingers on the final design. Rectangular and cylindrical features were tied into this design as well to give a 
simplified representation of the centered recessed chamber and the christmas-tree retainer features located at the bottom of the part. The modified design (Fig. 1b) incorporated the rounded outside corners, a recess located where the primary center heat of mass, as well as an angled side wall that was used to determine if there were any deflection. This design also added variable wall thickness as well as the tension finger that is located just below the main elbows where the curved fingers can be found. By removing material from the part design this reduced the overall volume of the mold cavity which in turn reduces that amount of heat transfer. The final mold design (Fig. 1c) was the final test part. This design included all complex features such as the tension fingers, variable wall thickness, and christmas-tree retainer. On all three mold inserts designs a $1.5^{\circ} \mathrm{draft}$ angle was used to help with manual ejection of the part from the mold inserts during actual testing.

Flow Analysis. This analysis was done to define the initial injection parameters for testing the designs. It was performed using the Moldflow software. The initial parameters that were observed from the Moldflow results were clamping force, temperatures upon injection, volumetric shrinkage, VP switchover, expected cooling times, and melt temperatures. The first analysis with Moldflow was also done to determine what should be expected from our part once injecting the designated material into the additively manufactured injection mold insert. The final analysis with Moldflow was done using our final injection parameters that were defined through the injection molding tests. The final analysis results verified the experimental results that were being observed, the temperature at mold open was upwards of $447^{\circ} \mathrm{C}\left(837^{\circ} \mathrm{F}\right)$ which was located in the exact locations were mold degradation occurred.

Finite Element Analysis. An FEA of the mold insert was performed to determine the maximum deflection around the part cavity. The maximum deflection would help to determine the amount of material needed to add to the overall height of the mold in order for the molds to completely seal when they were injected (to avoid flashing). The clamping force is calculated with the part and runner area, the cavity pressure, the separating force, and the factor of safety. The parameters and equations are as follows:

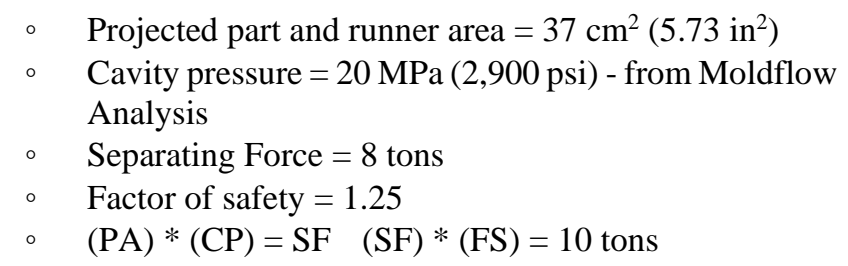

The FEA ran with 10 tons of clamping force on the front face of the mold, and 20 MPa of injection pressure on every face of the part cavity. The maximum deflection around the part cavity (Fig. 2) was $0.15 \mathrm{~mm}$. This meant an additional $0.15 \mathrm{~mm}$ to the overall height of our mold insert design.

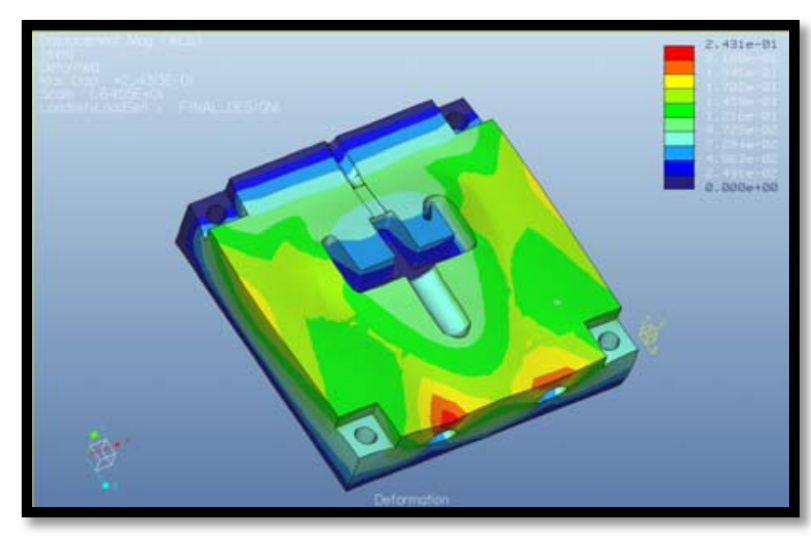

Figure 2. FEA Deflection Results.

After completing the final design, an FEA was run again to verify that the maximum deflection around the part cavity did not exceed $0.15 \mathrm{~mm}$ at the actual injection molding parameters used during testing [2]. For the final design the maximum deflection did not exceed $0.15 \mathrm{~mm}$.

Cooling Channel Design. The first step was to do heat transfer calculation based on the thermal properties on the 3DP insert (VeraGray) and the materials to be injected (Nylon and Polypropylene) to estimate the amount of heat energy being introduced to the mold inserts. Using the properties of cooling process water, and a static situation to simulate a worst case scenario, the volume the cooling channels needed to displace the generated heat was calculated. The objective was to define the channels' cross-sectional area. Unfortunately, these simplified calculations did not provide feasible dimensions. Polypropylene offered better results, but still unfeasible for our mold insert design. A second analysis was done taking into account the cooling pump and dynamic factors, producing a feasible design for polyprolpylene, with a $6.35 \mathrm{~mm}(0.25$ inches) circular cross section.

Taking advantage of using printed mold inserts, this first design for cooling channel is a "conformal" design, where the channel follows the profile of the cavity in a 2D path. A second design was used, where the channels follow a 3D path actually going under the center of mass of the part as well as having a rectangular cross section, where higher thermal mass is expected and therefore mold degradation was expected. In both designs analysis was done to structural integrity was ensured, and special access points were required to clean out the support material used for the channels during the printing process.

\section{RESULTS}

Testing of three different insert designs was performed using a Millacron 100-ton injection molding machine. The testing protocol was to conduct injection molding with pairs of mold inserts (Fig. 3), record parameters, inspect injected part (Fig. 4) and inserts, repeat until failure of the printed mold inserts. The results were as follows: 


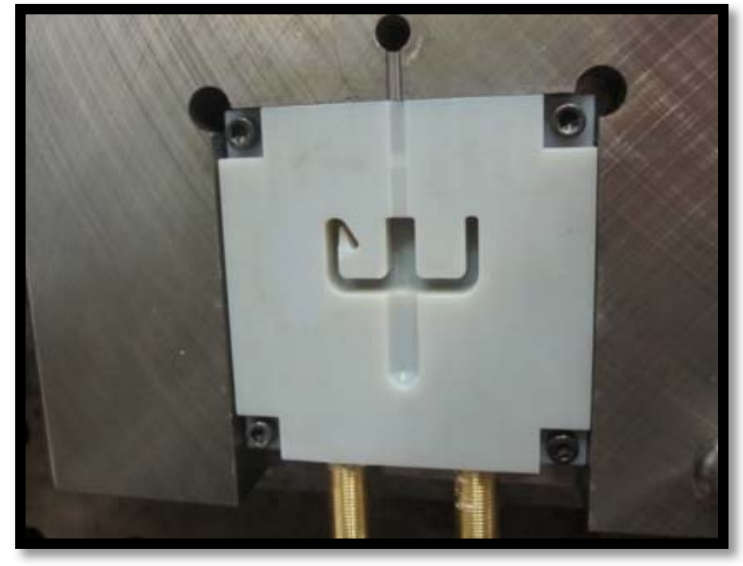

Figure 3. 3D Printed Half Insert on Injection Molding Machine.

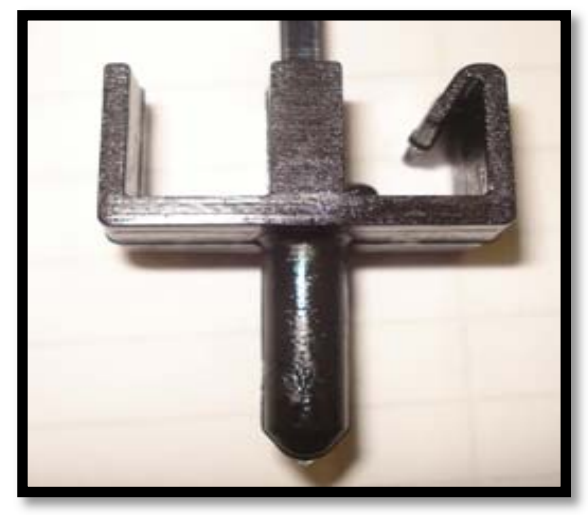

Figure 4. Injection Molded Part for Test Set 2.

Test Set 1. This set is for the initial design. Part material was both polypropylene and nylon. The mold inserts tested with polypropylene had cooling channels, and the mold inserts tested with nylon were tested with cooling channels and without cooling channels. Table I below shows that the inserts were able to take several shots, that the cooling channels have some effect, but few quality parts are obtained.

Test Set 2. This set is for the modified design. Polypropylene and nylon were used. In this test set polypropylene was tested without cooling channels, and nylon was tested with and without cooling channels. The table below shows a smaller number of acceptable parts, with inserts without channels performing better than with channels, for nylon.

Test Set 3. This set is for the final design. Polypropylene and nylon were used. In this test set there were no cooling channels. the table below shows that even a smaller number of shots were possible, with polypropylene giving the only quality part.

\section{DISCUSSION}

Throughout testing there were two materials that were primarily tested during injection molding. The main material tested was nylon, with 30 mold sets were tested, 14 with cooling and 16 without cooling. The average number of injection shots
TABLE I. RESULTS FOR SET TEST 1

\begin{tabular}{|c|c|c|c|}
\hline & Polypropylene & Nylon & Nylon \\
\hline Cooling & Yes & Yes & No \\
\hline \# Half & 6 & 26 & 10 \\
\hline \# Shots & 16 & 34 & 14 \\
\hline Quality & 1 & 2 & 4 \\
\hline
\end{tabular}

TABLE II. RESULTS FOR SET TEST 2

\begin{tabular}{|l|c|c|c|}
\hline & Polypropylene & Nylon & Nylon \\
\hline Cooling & No & Yes & No \\
\hline \# Half & 2 & 2 & 16 \\
\hline \# Shots & 6 & 4 & 23 \\
\hline Quality & 2 & 0 & 3 \\
\hline
\end{tabular}

TABLE III. RESULTS FOR SET TEST 3

\begin{tabular}{|l|c|c|}
\hline & Polypropylene & Nylon \\
\hline Cooling & No & No \\
\hline \# Half & 2 & 6 \\
\hline \# Shots & 10 & 3 \\
\hline Quality & 1 & 0 \\
\hline
\end{tabular}

obtained before failure was 2.42 with cooling, and 2.53 without cooling. This result of having more shots being performed with mold inserts that did not have cooling was due to the cooling lines having to be so close to the mold cavity that it affected negatively the integrity of the mold insert.

In testing with polypropylene a total of 5 mold insert sets were tested; 3 with cooling and 2 without cooling. The average number of injection shots obtained before failure was 4 with cooling, and 5 without cooling. The reason for fewer mold sets being tested is the average mold life with polypropylene is extended due to lower temperature of material melting point. The result of having more shots with inserts that did not have cooling was due to the cooling lines being too close to the mold cavity again which weakens the integrity of the material.

The two materials that were used exhibited different results from one another during injection. With nylon the cavity fills easily and transfer more feature details from the mold insert to the part. Due to the higher injection temperature of nylon, the 
insert would degrade after only one to two shots, with mold insert material melting and adhering to the part. Nylon also required longer cycle times to allow the insert to cool down in order to extend mold life. Polypropylene was capable of producing features well but was difficult to get to flow into more detailed areas. This issue was due to the higher injection viscosity of polypropylene which made setting the injection parameters more difficult. Lower cycle times with polypropylene were due to the lower injection temperature, and produce more parts per mold insert set with mold insert degrading after few shots. The criteria to consider an injected part as a quality (i.e., acceptable) part were: a) no visual defects in the injected part, b) specified tolerances, and c) acceptable surface finish. The mold inserts were deemed unusable when they had experienced enough degradation.

\section{CONCLUSIONS AND RECOMMENDATIONS}

Upon completion of this study it was concluded that the use of AM techniques to manufacture injection mold inserts is feasible. There was evidence that AM mold inserts have the potential to serve for short run production of functional prototypes for rapid quoting. The test part has geometric features that are common in plastic fasteners, and quality parts were realized for two materials. Throughout the testing it was possible to determine that the mold material VeroGrey was able to withstand the clamping and injection pressures required for injection molding, and there is a new material VeroGreen that has higher heat deflection temperature, which is expected to improve the thermal life of the printed part.

Based on the results from testing it was concluded that in our test sets cooling had no significant impact on mold insert life or number of quality parts produced. Due to the low thermal conductivity of the printed material, for cooling to be effective at removing heat the channel needed to be too close to the part surface, causing a thin wall between the mold cavity and the cooling channel. This thin wall would then crack under the parameters for injection molding, causing the cooling channels to become filled with injection material, thus destroying the mold inserts.

The following recommendations are made:

a) to have exact material properties, this is of vital importance for a project of this nature. In order to have valid prediction of performance there is need to have reliable properties

b) redesign inserts to accommodate cooling channels, the structural integrity after thermal fatigue is believed to be the main cause of insert failure. Cooling channels should help in the process, not be a liability.

\section{REFERENCES}

[1] 3D Printing. (n.d.). Retrieved 7 14, 2015, from Wikipedia: http://en.wikipedia.org/wiki/Additive_manufacturing

[2] Stratasys 3D Printers. (2015, 6 5). New Objet Materials: The power behind your 3D Printer. Retrieved 2015, from
http://www.objet.com/Portals/0/docs2/New\%20materials\%20data\%20sh eets_low\%20res.pdf

[3] DLG. (2012). Digital Lab Graph. Retrieved Feb. 12, 2014, from http://factoryoffactories.com/rapidtool.htm

[4] PolyJet Materials Data Sheet. (n.d.). Retrieved 7 8, 2013, from http://www.stratasys.com/materials/polyjet/ /media/29592222B80C489 BAC28803DB08C10E5.ashx

[5] Center for Integrated Design.

[6] Zheng, T. a. (2011). Injection Molding: Integration of Theory and Modeling Methods. Longdon, New York: Springer Heidelberg Dordrecht.

[7] Dickens, P. (2000). Failure Mechanism Analsyis of Stereolithographic Injection Mould Tooling. Rapid Prototyping Journal, 62-74.

[8] Jacobs, P. F. (1996). Recent advances in rapid tooling for Stereolithography. Proceedings of the national conference on Rapid Prototyping and Tooling Research (pp. 18-19). Buckinghamshire: Buckinghamshire College.

[9] Jacobs, P. F., \& Hilton, P. D. (2000). Rapid Tooling: Technologies and Industrial Applications. In P. F. Peter D. Hilton. New York: Marcel Dekker.

[10] Nagahanumaiah, Subburaj, \& Ravi. (2008). Computer aided rapid tooling process selection and manufacturability evaluation for injection mold development. Computers in Industry, 59(2-3), 262-276.

[11] http://www.stratasys.com/resources/case-studies/education/budapestuniversity-of-technology-and-economics. Budapest University of Technology and Economics, 3D printing helps university create functional injection molded prototypes for testing. Dr. József Gábor Kovács,

[12] 3DP Injection Molding Process Spread by Stratasys and Worrell Collaboration. http://3dprintingindustry.com/2014/11/05/injectionmolding-stratasys-worrell/

$14^{\text {th }}$ LACCEI International Multi-Conference for Engineering, Education, and Technology: "Engineering Innovations for 